\title{
Interference Aware Bluetooth Scatternet (Re)configuration Algorithm IBLUEREA
}

\author{
Tomasz Klajbor ${ }^{1}$, Jozef Wozniak ${ }^{1}$ \\ ${ }^{1}$ Gdansk University of Technology, Faculty of Electronics, \\ Telecommunications and Informatics, Gdansk, Poland \\ \{klajbor, jowoz\}@pg.gda.pl
}

\begin{abstract}
This paper presents a new algorithm IBLUEREA, which enables the reconfiguration of Bluetooth scatternet to reduce interference. IBLUEREA makes use of the complex model comparing ISM environment efficiency. The mechanism envisages the use of the assessment of the probability of successful (unsuccessful) frame transmission in order to take a decision concerning the coexistence of technologies which make use of the same ISM band (here Bluetooth and $802.11 \mathrm{~b}$ ).
\end{abstract}

Keywords: Bluetooth, IEEE 802.11b, interference, co-existence

\section{Introduction}

The number of various wireless technologies and network devices making use of ISM band (e.g. Bluetooth (BT) 2, IEEE 802.11b (Wi-Fi) 7 or IEEE 802.11g) is growing very fast. Due to this, it becomes more and more difficult to provide transmission parameters that can guarantee the quality of services required by coexisting networks. This specially refers to specific network devices operating in a close vicinity around other devices belonging to different independent networks, very often based on different technical and functional solutions.

In order to provide for a higher work efficiency of a number of technological solutions working within the same area, coexistence mechanisms have been worked out 1 . Such mechanisms can be divided into two groups 1:

- Collaborative mechanisms, requiring information exchange between IEEE $802.11 \mathrm{~b}$ and Bluetooth devices.

- Non-collaborative mechanisms, which can be adopted by $802.11 \mathrm{~b}$ and/or Bluetooth devices without a direct collaborative system.

Apart from the mechanisms presented in 1, examples of different collaborative algorithms can be found in the literature. Isolated examples of solutions facilitating the co-existence of various technologies can be traced, which are based on predicting the propagation conditions variability. For example, in 4 Interference aware BLUEtooth Segmentation mechanism has been presented which is based upon a dynamic BT frame choice depending on the propagation conditions. This method relies upon the theoretical assessment of the probability of successful frames transmission and the queuing tasks analysis. Based on such information IBLUES

Please use the following format when citing this chapter:

Klajbor, T., Wozniak, J., 2007, in IFIP International Federation for Information Processing, Volume 245, Personal

Wireless Communications, eds. Simak, B., Bestak, R., Kozowska, E., (Boston: Springer), pp. 145-156. 
"takes" decisions concerning the choice of a frame (from those defined in specification 2), through which the data will be transferred (e.g. DM1, DM3, DM5).

In this article a new coexistence mechanism has been presented which is correlated to the management of Bluetooth network topology. This mechanism has been named Interference Aware BLUEtooth Scatternet (RE)configuration Algorithm (IBLUEREA). IBLUEREA algorithm is based upon the idea of switching functions performed by those BT devices which more frequently use ISM band (i.e. masters) and are in close vicinity of receivers/transmitters of other technology solutions (e.g. $802.11 \mathrm{~b}$ ) and BT piconets. IBLUEREA involves operating as a master (in a given piconet) for a device which simultaneously causes and is susceptible to little interference (comparing to other BT piconet devices and networks using other technologies). For this analysis, IBLUEREA uses a new model comparing the efficiency of ISM complex environment.

Chapter 2 describes the above-mentioned model comparing the efficiency of ISM environment. Theoretical grounds of this model have been equipped with a simple example illustrating its mechanism.

Chapter 3 presents the idea behind the IBLUEREA algorithm. Chapter 4, in turn, presents the benefits of using this algorithm by giving scenarios, which has been the subject of simulations tests.

\section{Model comparing the efficiency of ISM environment}

While making comparisons regarding the efficiency of given ISM environments, among others, the number and function of various technology devices which are colocated need to be taken into consideration. In order to assess with accuracy the efficiency of a given ISM environment, the influence of all jamming devices on the receivers located within their range need to be accounted for.

In 6 the general principles of scatternet matrix description have been presented. The authors also suggested metrics (allowing for functions played by devices in given piconet and/or devices links), thanks to which the aggregated (and standardized) link capacity in scatternet assessment is possible. The metrics are of little significance while tackling the interference issue, which has only been mentioned in this article. Moreover, metrics do not enable the analyses of interference coming from other systems. Below, it has been presented the original methodology and key metrics necessary for the Bluetooth scatternet and IEEE $802.11 \mathrm{~b}$ network co-effectiveness assessment.

Let us assume that a scatternet with the topology presented in Fig. 1 has been created. Devices 1 and 4 operate as masters. Piconet 1 includes slave devices with numbers 2 and 3 . Whereas piconet 2 makes slave devices 3 and 5. Slave device no. 3 acts as a bridge between the two piconets. 


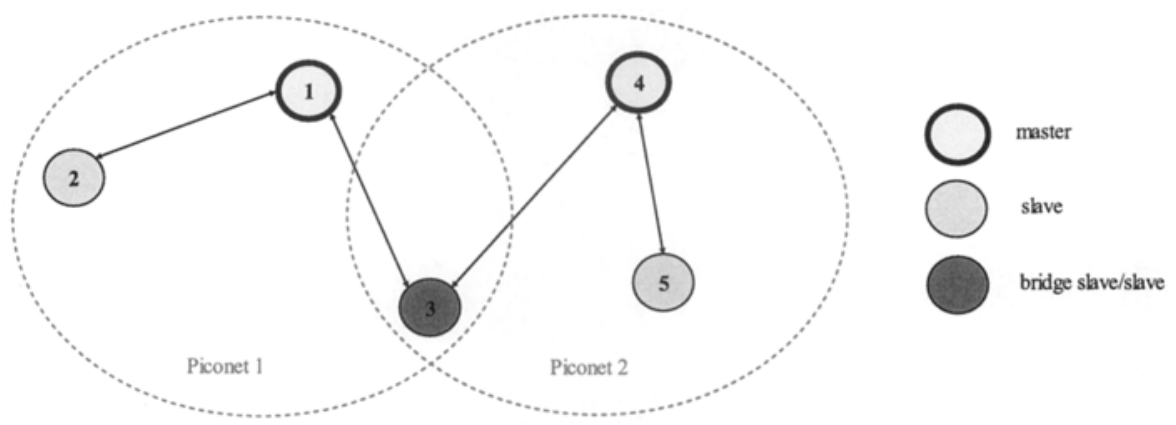

Fig. 1. An example of scatternet consisting of two BT piconets.

In order to illustrate co-existence mechanism, Fig. 2 presents also an example of ISM environment, with possible mutual interference areas of the Bluetooth piconet and the coexisting IEEE $802.1 \mathrm{lb}$ network. It has been assumed that the mutual interference areas are those where given technology transmitters have a substantial negative impact on the receivers of other BT piconets or $802.1 \mathrm{lb}$ network (for example, frame error rate can, in theory, exceed a given threshold value).

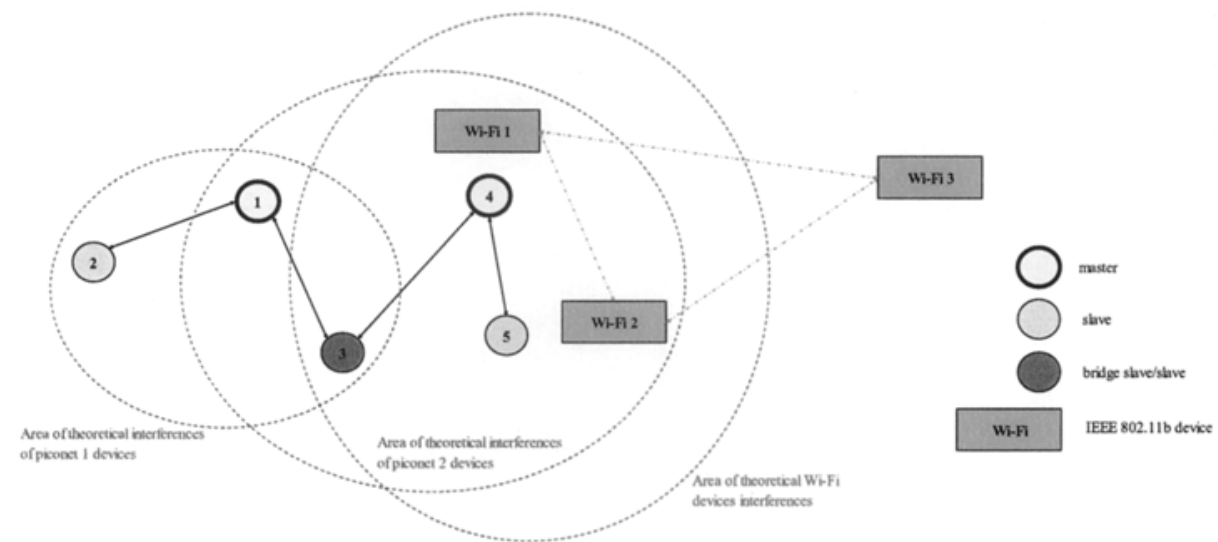

Fig. 2. Example of Bluetooth and Wi-Fi mutual interference areas

In accordance with the above illustration:

- Piconet 1 can potentially jam the transmission of device 3 , in case when it operates as an element of $2^{\mathrm{l}}$ piconet,

- Piconet 2 jams the transmissions between 1 and 3 devices (piconet 1 ) and jams the receptions at IEEE $802.11 \mathrm{~b}$ : Wi-Fi 1 and Wi-Fi 2,

- IEEE 802.11b network jams the transmissions of BT: 3, 4 i 5 ,

- Wi-Fi 3 is beyond interference over BT scatternet.

1 To simplify the analyses, it has been assumed that if a device belongs to a given piconetwork, it should affect the interference under examination. In fact, the interference range of each individual device with another one should be examined. 
Such an ISM environment, defined in such way, can be formally presented using the interference matrix $\mathbf{A}_{(\mathrm{B}+\mathrm{W}) \times(\mathrm{B}+\mathrm{W})}$ :

$$
A_{(B+W) \times(B+W)}=\left[\begin{array}{llllllll}
0 & 0 & 1 & 0 & 0 & 0 & 0 & 0 \\
0 & 0 & 1 & 0 & 0 & 0 & 0 & 0 \\
1 & 0 & 0 & 1 & 1 & 1 & 1 & 0 \\
1 & 0 & 1 & 0 & 0 & 1 & 1 & 0 \\
1 & 0 & 1 & 0 & 0 & 1 & 1 & 0 \\
0 & 0 & 1 & 1 & 1 & 0 & 0 & 0 \\
0 & 0 & 1 & 1 & 1 & 0 & 0 & 0 \\
0 & 0 & 0 & 0 & 0 & 0 & 0 & 0
\end{array}\right]
$$

where B - the number of BT scatternet devices; $\mathrm{W}$ - the number of Wi-Fi network devices.

Columns of matrix A represent subsequent (as per numerical description): BT scatternet devices, and following IEEE $802.11 \mathrm{~b}$ network devices. The elements of the matrix of $\mathrm{a}_{\mathrm{i}, \mathrm{j}}$ are created in the following way: if a given $i$ device potentially jams $j$ device, then $\mathrm{a}_{\mathrm{i}, \mathrm{j}}$ equals 1 (otherwise 0 ). Therefore, $i$ lines of matrix $\mathbf{A}$ represent jamming devices, whereas columns $j$ - the devices being jammed.

Matrix A presented in (1), informs which devices can cause interferences with other.

Let's mark additionally with: $m$ - the master group, $s$ - slave group (responding to given masters) and $b$-group of bridge devices for a given scatternet. For the network presented in Fig. 2, those sets are: $m=\{1 ; 4\}, s=\{(2,3) ;(3,5)\}$ and $b=\{3\}$.

Let's mark by $p_{i}$ a set of all devices of the Bluetooth piconet, within which the $i$ device operates.

To estimate the impact of each $i$ jamming device over $j$ we will create matrix $\mathbf{X}$. This matrix specifies the theoretical jamming frequency of $j$ devices by $i$ devices. Let us also assume that all devices have queued tasks (traffic load $=1$ ). The mode of setting $\mathrm{x}_{\mathrm{i}, \mathrm{j}}$ elemnets of $\mathbf{X}$ matrix has been presented below.

Each master device of $i \in \mathrm{m}\left(i \in p_{i}\right)$ jamming a given device of $j \in p_{j}$, as per the above assumptions, manages $p_{i}$ piconet creating every second frame, therefore:

$$
\underset{a_{i, j} \neq 0}{\forall} \underset{i \in m}{\forall} j \in p_{j} \Rightarrow x_{i, j}=\frac{a_{i, j}}{2}=\frac{1}{2}
$$

Whereas the slave device of $i \in \mathrm{s}\left(i \in p_{i}\right)$, interferes other devices at the frequency of calling up such device within $p_{i}$ piconet, that is:

$$
\underset{a_{i, j} \neq 0}{\forall} \underset{i \in s}{\forall} j \in p_{j} \Rightarrow x_{i, j}=\frac{1}{2 \cdot s_{i}}
$$

where $s_{i}$ means the number of slave devices within $p_{i}$ piconet.

For devices connecting Bluetooth networks $i \in b$ we assume formula (2), remembering that the bridge device can interfere all networks it links, and within 
which it is not currently performing transmission. For devices of other technologies (here $802.11 \mathrm{~b}$ ) we assume the maximum value under formula (2).

For the devices operating within $802.11 \mathrm{~b}$ network we assume that the rival access to the medium requires the use of ISM band:

$$
\underset{i, j \notin m \cup s}{\forall} x_{i, j}=\frac{a_{i, j}}{L_{i, j}}
$$

where: $L_{i, j}-$ number of IEEE $82.11 \mathrm{~b}$ devices in a given Wi-Fi network ${ }^{2}$.

The above quoted formulas (2) - (3) relate directly to a BT scatternet, whereas (4) to IEEE $802.11 \mathrm{~b}$ network. Using the relations form (2) to (4), matrix $\mathbf{A}$ can be modified, which now transforms to matrix $\mathbf{X}$, presented as (5).

$$
X_{(B+W) \times(B+W)}=\left[\begin{array}{cccccccc}
0 & 0 & 1 / 2 & 0 & 0 & 0 & 0 & 0 \\
0 & 0 & 1 / 4 & 0 & 0 & 0 & 0 & 0 \\
1 / 4 & 0 & 0 & 1 / 4 & 1 / 4 & 1 / 4 & 1 / 4 & 0 \\
1 / 2 & 0 & 1 / 2 & 0 & 0 & 1 / 2 & 1 / 2 & 0 \\
1 / 4 & 0 & 1 / 4 & 0 & 0 & 1 / 4 & 1 / 4 & 0 \\
0 & 0 & 1 / 3 & 1 / 3 & 1 / 3 & 0 & 0 & 0 \\
0 & 0 & 1 / 3 & 1 / 3 & 1 / 3 & 0 & 0 & 0 \\
0 & 0 & 0 & 0 & 0 & 0 & 0 & 0
\end{array}\right]
$$

Each of the possible interferences of $a_{i, j}$ (or respectively $x_{i, j}$ ) features a given frame error rate, which can be specified for each situation under analysis. Therefore, it is possible to create the matrix $\mathbf{B}_{(\mathrm{B}+\mathrm{W}) \times(\mathrm{B}+\mathrm{W})}$ - successful frame transmission probability matrix (at the entry of given $j$ receiver). The elements of matrix $\mathbf{B}$ have been described in the following way:

$$
b_{i, j}=x_{i, j} \cdot P_{S(1, j)}
$$

where:

$\mathrm{P}_{\mathrm{S}(\mathrm{i}, \mathrm{j})}$ - The probability of a successful frame reception by $j$ device, allowing for a potential interference from $i$ device (and other propagation conditions ${ }^{3}$.

2 Just assumption for all Wi-Fi devices, in particular those whose transmission do not affect Bluetooth scatternet efficiency (that is located in a significant distance from BT devices), but using ISM band within a given BSS.

3 Considering only a bit error rate would not allow for the dynamic ISM environment changes and the co-existence of various technologies (especially frame collision probability on the frequency level). 
The probability of $\mathrm{P}_{\mathrm{S}(\mathrm{i}, \mathrm{i})}$ can be in general written as follows:

$$
P_{S(i, j)}=\sum_{N} P_{S}\left(P_{E} \mid n\right) \cdot P_{C}(n, N)
$$

where:

$P_{C}(n, N)$ - probability of given technology frame collision with $n$ other technology frames (or the same technology for Bluetooth piconet) out of $N$ possible collisions (frequency analysis),

$P_{S}\left(P_{E} \mid n\right)$ - respectively the probability of a successful reception of IEEE $802.11 \mathrm{~b}$ frame(Bluetooth), which was subject, or was not, of a collision (time analysis).

Within $P_{S}\left(P_{E} \mid n\right)$ probability it is important to specify the bite error rate of $\mathrm{P}_{\mathrm{E}}$ in case of a collision and in lack of collision. The relations of the bite error rates have been presented in 1 .

For such created matrix $\mathbf{B}$ its metric $\beta$ can be determined, which represents standard average efficiency measure of coexisting networks:

$$
\beta=\frac{1}{2} \alpha_{1}+\frac{1}{2} \alpha_{2}
$$

where:

- $\boldsymbol{\alpha}_{1}-$ standardized BT network efficiency measure:

$$
\alpha_{\mathrm{1}}=\frac{\sum_{i=1}^{B} \sum_{j=1}^{B} b_{i, j}}{\sum_{i=1}^{B} \sum_{j=1}^{B} a_{i, j}}
$$

- $\quad \alpha_{2}-$ standardized IEEE $802.11 \mathrm{~b}$ network efficiency measure :

$$
\alpha_{2}=\frac{\sum_{i=B+1}^{B+W} \sum_{j=B+1}^{B+W} b_{i, j}}{\sum_{i=B+1}^{B+W} \sum_{j=B+1}^{B+W} a_{i, j}}
$$

Measure $\beta$ is a numerical representation of the standardized sum of interference frequency affecting the devices within a given network (given technology solution), simultaneously allowing for the successful transmission probability (despite such interference) Having parameter $\beta$ it is possible to specify the efficiency of the coexisting BT and IEEE $802.11 \mathrm{~b}$ networks (and not only) as far as their mutual interferences are concerned. Metric $\beta$ is this way a measure for comparing the efficiency of the complex ISM ${ }^{4}$ environments. The lower the value of $\beta \geq 0$, the lower

4 A constraint of this method is the lack of allowing for the traffic analysis. In order to assess in with a greater accuracy this method can be extended by elements of e.g. the link average capacity within given network. For the purposes of the study of interference ratio, the analysis has been focused upon the model aspects irrespective of the traffic generated within given networks. 
the interference ratio (mutual jamming) of a given ISM environment by various technologies. Optimal ISM environment is a such one which could be specified with a minimum measure $\beta$ (criterion function).

Based upon the above described ISM environment efficiency comparison method, the reconfiguration (creation) of co-located networks topology is possible which would provide for the lowest mutual interference ratio. The algorithm enabling such function has been presented in the following chapter.

\section{Nature of IBLUEREA algorithm}

IBLUEREA algorithm, which is based upon the above described ISM environment efficiency method, is a suggests a reconstruction mechanism for Bluetooth scatternet. The idea behind IBLUEREA, relies upon the changes of functions fulfilled by a device (master, slave, bridge). IBLUEREA decisions are taken upon ISM environment features (the above described criterion function), which encompasses the analysis of potential interferences coming from different transmitters.

IBLUEREA algorithm encompasses the following operations cycle, preceded by the use of a mechanism generating scatternet of a minimal number of piconet ${ }^{5}$ :

1. BT master devices control the traffic load of ISM ${ }^{6}$ band and have information on the number of piconets within a given scatternet,

2. At spotting complex $\mathrm{ISM}^{7}$ environment, BT master devices appoint randomly from them an IBLUEREA coordinator.

3. The coordinator triggers the procedure of establishing parameter $\beta_{l}$, that is a standardized efficiency of coexisting networks (for the existing topology), simultaneously assessing the network reconfiguration possibility to provide for the lowest possible interference ratio $\left(\beta_{2}\right.$ for a new topology); information for the coordinator come from BT devices, which assess the frame successful transmission probability.

4. Upon collecting information from all $\mathrm{BT}$ master devices and the devices of other technologies (here Wi-Fi), the coordinator takes a decision on a possible network reconfiguration as per the new functions assigned.

IBLUEREA operating mechanism has been presented in Fig. 3 as a simple sequence of operations.

e.g. Law, Mehta and Siu mechanism 5.

The band control can be done e.g. every 30 time slot.

Complex ISM environment is such which, for example, includes a number of piconets and the level of potential $\mathrm{Wi}-\mathrm{Fi}$ interferences exceeds given threshold value. 


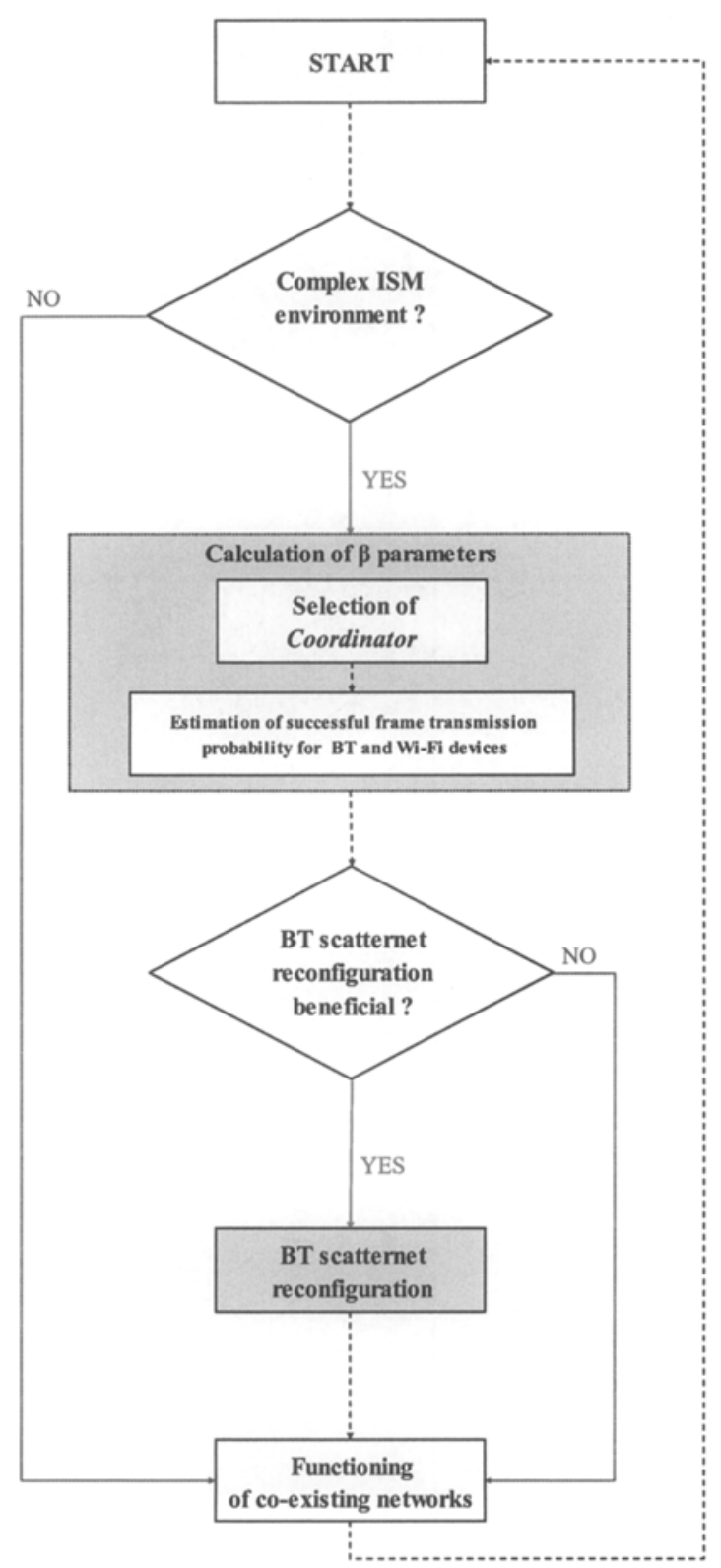

Fig. 3. IBLUEREA algorithm.

Each Bluetooth piconet is a subject of reconfiguration in order to provide for a function for such master device which is logically located as far as possible from the intererence source and/or can be in the least a source of interferation itself (here $802.11 \mathrm{~b}$ network or other BT piconets). The coordinator appoints the new device upon a BT frame successful transmission probability (within a given piconet). The 
decision of the scatternet reconfiguration takes place when the minimal (lower ${ }^{8}$ ) parameter $\beta$ is found (in relation to measure, $\beta$, ressulting from the current ISM environment).

To illustrate the algorithm mechanisms, examples of its functioning have presented further on (see Fig. 4).

\section{Example of IBLUEREA functioning}

In order to assess IBLUEREA usefulness, let us consider model operating scenarios of Bluetooth and $802.11 \mathrm{~b}$ networks.

Fig. 4 gives examples of to theoretical topologies. The first is a result of a BT scatternet exemplary formulating. The letter case envisages the use of IBLUEREA algorithm for Bluetooth network reconfiguration. Fig. 4 includes some simplifed assmptions regarding network impact range and allowing for a better presentation of the mechanism itself.
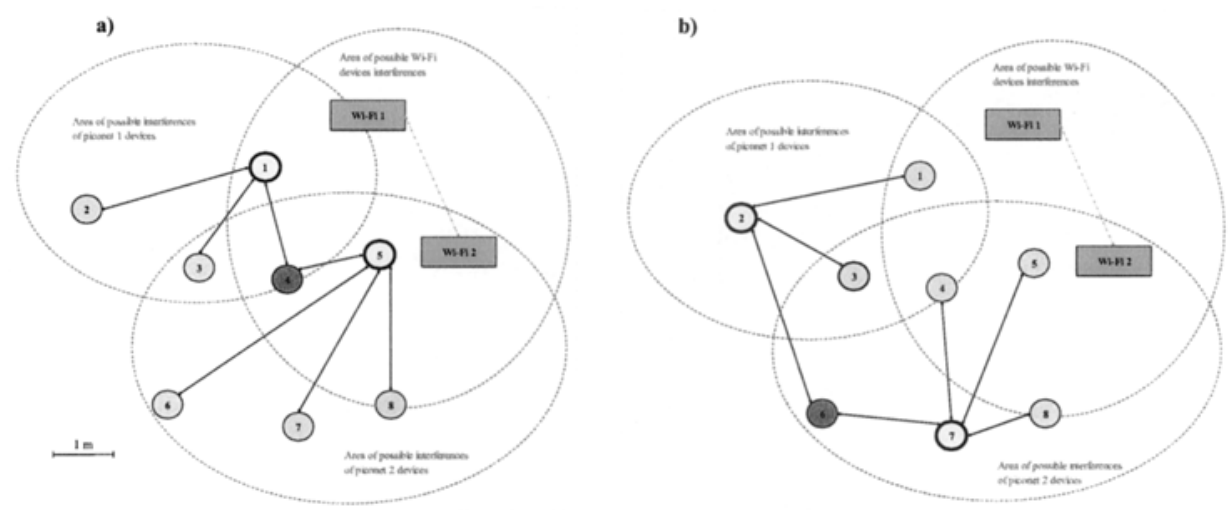

Fig. 4. Illustration of functioning of IBLUEREA algorithm: a) topology before reconfiguration, b) topology after IBLUEREA algorithm effecting changes.

Let us assume that the subject of our study are frames $1500 \mathrm{~B}$ long for $802.11 \mathrm{~b}$ and frames DH1 for Bluetooth generated by constant bit rate source. By establishing parameters $\beta$ for the quoted scenarios $w$ receive respectively: $\beta_{a}=0,26>\beta_{b}=0,21$. Measure $\beta_{b}$ simultaneously specifies the minimum value of the parameter to be obtained in a such ISM environment (under the accepted comparison model).

The calculation of parameter $\beta$ is necessary for taking the decision whether the suggested reconfiguration is likely to bring about expected benefits of ISM band efficiency enhancement through colocated network or not. It could be especially useful for highly complex ISM environments, in which in a relatively close vicinity coexits a nmber of various technologies and devices.

8 Considerations concerning the search for only lower measures $\beta$ can be a result of the implementation of IBLUEREA algorithm itself. 
To illustrate the effect of IBLUEREA mechanism exemplary results of studies performed by way of simulations for a complex ISM environment (which allows for without limitations, the structure from fig. 4$)^{9}$ have been presented below.

Table 1 presents the results of simulation measurements for four cases (example 1). The coexistence of BT and $802.11 \mathrm{~b}$ network was analysed which did not have coexistence mechanism triggered (FH - Frequency hopping BT) and using the AFH mechanism 1 (Adaptive Frequency Hopping) - see Fig. 4A. For the purposes of comparison a situation was analysed in which the topology was reconfigured as a result of IBLUEREA algorithm functioning (which corresponds with Fig. 4B).

Tab. 1. Average frame error rate of Bluetooth (class II devices) and $802.11 \mathrm{~b}$ networks (example 1)

\begin{tabular}{|c|c|c|c|c|}
\cline { 2 - 5 } \multicolumn{1}{c|}{} & \multicolumn{4}{c|}{ Mechanism used } \\
\cline { 2 - 5 } \multicolumn{1}{c|}{} & $\mathrm{FH}$ & $\mathrm{AFH}^{10}$ & $\begin{array}{c}\mathrm{FH}+ \\
\text { IBLUEREA }\end{array}$ & $\begin{array}{c}\text { AFH }+ \\
\text { IBLUEREA }\end{array}$ \\
\hline $\begin{array}{c}\text { Average } \\
\text { BT FER [\%] }\end{array}$ & 57.0 & 3.9 & 56.8 & 2.4 \\
\hline $\begin{array}{c}\text { Average } \\
\text { 802.11 FER [\%] }\end{array}$ & 20.5 & 0.0 & 9.3 & 0.0 \\
\hline
\end{tabular}

As presented in Tab. 1, making use of IBLUEREA mechanism (upon the analysed example) facilitates the efficiency of coexisting Bluetooth and $802.11 \mathrm{~b}$ networks both for the scenario where no coexistence mechanism was used $(\mathrm{FH})$, and where Bluetooh network triggered the AFH mechanism. As presented in the example under analysis, the band efficiency with triggered AFH was increased. It needs to be stressed out that in a situation where in a close viccinity function various technology solutions, using various transmission modes, often without one permanent band, the use of AFH algorithm can be hindered. Moreover, studies show that AFH algorithm is relatively inefficent for dynamic ISM environment changes, in which the same frequence is used for a short period of time.

To illustrate the benefits of IBLUEREA algorithm also using AFH algorithm let us analyse a more complex ISM environment (example 2). Let us assume that the ISM environment in question consists of 3 independent $802.11 \mathrm{~b}$ networks and 4 BT piconets (20 class II devices) scattered over the radius of $10 \mathrm{~m}$. We are examining two scenarios: in the first master devices are equally located within $4 \mathrm{~m}$ from the excentre and in the letter within $9 \mathrm{~m}$. Tab. 2 compares ISM environment efficiency using AFH mode.

Tab. 2. Average frame error rate of Bluetooth (class II devices) and $802.11 \mathrm{~b}$ networks (example 2)

\begin{tabular}{|c|c|c|}
\cline { 2 - 3 } \multicolumn{1}{c|}{} & \multicolumn{2}{c|}{ Mechanism used } \\
\cline { 2 - 3 } \multicolumn{1}{c|}{} & AFH & AFH + IBLUEREA \\
\hline Average BT FER [\%] & 12.5 & 4.0 \\
\hline Average 802.11 FER [\%] & 0 & 0 \\
\hline
\end{tabular}

9 It has been additionally assumed that $802.11 \mathrm{~b}$ use two channels to enhance their throughput. It corresponds with the two independent Wi-Fi networks scenario.

10 For simplicity reasons, it has been assumed that only channels used by $802.11 \mathrm{~b}$ network are avoided. Therefore mutual interferences only from BT piconets are possible. 
In the scenarios from the second example, in fact, the issue relating the mutual BT piconet is being examined. As shown in Tab. 2, making use of IBLUEREA algorithm can have a positive impact on the enhancement of ISM band efficiency through the use of AFH devices. It can be particularily useful for sensor networks comprising of a large number of Bluetooth devices.

\section{Summary}

This paper presents a new IBLUERA control mechanism based upon the use of a new model comparing the efficiency of ISM environment. It has been proved that IBLUEREA can facilitate the coexistence mechanisms in use (including AFH mechanism 1).

An advantage of IBLUEREA algorithm is its potential to reduce mutual interferences of BT and $802.11 \mathrm{~b}$ networks. It needs to be stressed out that IBLUEREA algorithm can be implemented togehter with other mechanisms (e.g. Law, Mehta and Siu algorithm 5), even at the BT scatternet formation stage. A disadvantage of such algorithm is the necessity of information exchange between coexisting technologis and additional LMP frames exchange (in Bluetooth). Moreover BT network needs to allow for the time slot to monitor the ISM band usage.

In future works, the authors plan to compare IBLUEREA mechanism while using chosen algorithms for Bluetooth network formation.

\section{References}

1. IEEE, IEEE Std 802.15.2-2003, IEEE Recommended Practice for Information technology - Telecommunications and information exchange between systems - Local and metropolitan area networks - Specific requirements. Part 15.2: Coexistence of Wireless Personal Area Networks with Other Wireless Devices Operating in Unlicensed Frequency Bands, August 2003.

2. Bluetooth SIG, Inc., Specification of the Bluetooth System, Covered Core Package version: $2.0+E D R, 4$ November 2004, http://www.bluetooth.org.

3. Klajbor, T.; Jaszcza, A.; Effectiveness of IEEE802.11b system in neighbourhood of Bluetooth piconet (in Polish), Proceedings of $3^{\mathrm{RD}}$ Conference on Information Technology, Gdańsk University of Technology, Faculty of Electronics, Telecommunications and Informatics, May 2005, Volume 7, pp. 369-376.

4. Cordeiro, C.D.M. Agrawal, D.P., Employing dynamic segmentation for effective colocated coexistence between Bluetooth and IEEE 802.11 WLANs, IEEE Global Telecommunications Conference, 2002. GLOBECOM '02., 17-21 Nov. 2002, Volume: 1, page(s): 195- 200.

5. C. Law, A. K. Mehta, and K.-Y. Siu, A new Bluetooth scatternet formation protocol, Mobile Networks and Applications, vol. 8, no. 5, Oct. 2003.

6. Cuomo, F.; Melodia, T.; A general methodology and key metrics for scatternet formation in Bluetooth, IEEE Global Telecommunications Conference, 2002. GLOBECOM '02. Volume 1, 17-21 Nov. 2002 Page(s): 941 - 945 vol.1. 
156 PWC 2007

7. IEEE, IEEE Std $802.11 \mathrm{~b}-1999$ (R2003), Supplement to IEEE Standard for Information technology - Telecommunications and information exchange between systems - Local and metropolitan area networks - Specific requirements - Part 11: Wireless LAN Medium Access Control (MAC) and Physical Layer (PHY) specifications: Higher-Speed Physical Layer Extension in the 2.4 GHz Band, New York, June 2003.

8. Golmie, N.; Rebala, O.; Chevrollier, N.; Bluetooth adaptive frequency hopping and scheduling, IEEE Military Communications Conference, 2003. MILCOM 2003., Volume 2, 13-16 Oct. 2003 pp.:1138 - 1142 Vol.2 